Bull. Mater. Sci., Vol. 36, No. 6, November 2013, pp. 981-987. (c) Indian Academy of Sciences.

\title{
Green chemical incorporation of sulphate into polyoxoanions of molybdenum to nano level
}

\author{
JADU SAMUEL*, S HARI PRASAD and M K SREEDHAR ${ }^{\dagger}$ \\ Department of Chemistry, Mar Ivanios College, Thiruvananthapuram 695015, India \\ ${ }^{\dagger}$ Department of Chemistry, University College, Thiruvananthapuram 695034, India
}

MS received 19 June 2012; revised 25 September 2012

\begin{abstract}
Synthesis of sulphomolybdate (SMO) nanospheres was carried out by using sodium molybdate and a structure directing cationic surfactant, dodecyl pyridinium chloride (DPC) at room temperature by applying green chemistry principles. The composition and morphology of the nanospheres were established by Fourier transform infrared spectroscopy (FT-IR), scanning electron microscopy (SEM), transmission electron microscopy (TEM), atomic force microscopy (AFM), thermogravimetric analysis (TGA) and inductively coupled plasma atomic emission spectroscopic (ICP-AES) techniques. Nanospheres in the diameter range of 10-100 $\mathrm{nm}$, having the highest population within $50 \mathrm{~nm}$ were obtained. The effects of reaction parameters such as concentration of surfactant, temperature and $\mathrm{pH}$ of solution on the synthesis of nanospheres were investigated. The mechanism of agglomeration of nanoparticles are also discussed.
\end{abstract}

Keywords. Polyoxometalate; green synthesis; nanostructures; surfactant.

\section{Introduction}

As our living standard is rapidly improving and the pace of industrialization is accelerating, the environmental pollution is becoming a serious problem. To combine the technological progress with environmental safety is one of the key challenges of the millennium. In the efforts to move towards 'sustainable development', chemistry is, now-a-days at the forefront of development of clean production process and products (Sanghi and Srivastava 2007). 'Green chemistry' is a science-based non-regulatory and economically driven approach for achieving the goals of environmental protection and sustainable development. In order to be eco-friendly, green chemical synthesis must meet the requirements such as absence of waste, atom efficiency, avoid the use and production of toxic and dangerous chemicals; reduce energy requirements and use of catalysts rather than stoichiometric reagents.

The versatile nature of polyoxometalates (POM) in terms of their structure, size, redox chemistry, photochemistry and charge distribution means that it is arguably one of the main areas in inorganic chemistry that is developing most rapidly today (Long et al 2007). New areas are emerging in POM chemistry that are multidisciplinary and exploit great structural and electronic diversity of POM-based systems. Much of the interest in these molecules has arisen because such clusters represent a paradigm in the discovery of systems that can be encouraged to grow from molecular to the nanoscale (Liu et al 2003). In terms of forming direct POM

\footnotetext{
*Author for correspondence (prof_jadu@ hotmail.com)
}

polymers linked by Mo-O-Mo units, it has been observed that in the synthesis of wheel- and sphere-shaped nanosized molybdenum oxide-based clusters even under one-pot conditions, extensive linking via these types of units can occur. This means that the clusters primarily formed by self assembly can become further linked in the same phase (Muller et al 2002). An example is that the paramagnetic Keplerate 'necklaces' synthesized by a novel room temperature solid-state reaction were characterized in the solid state as metal oxidebased nanoparticles which are formed from controlled linking of $\left\{\left(M_{\mathrm{o}}^{\mathrm{VI}}\right)-M_{\mathrm{O}}^{\mathrm{VI}}\right\}_{12} \mathrm{Fe}_{30}$ type Keplarate balls connected by inter-ball Mo-O-Mo bonds.

Peng's group (Kang et al 2005) reported a series of work on $\left\{\mathrm{Mo}_{6}\right\}$ imine compounds in which POMs are covalently bonded with terpyridine ligands, which can coordinate to other metal ions to form coordination polymers and a rational synthesis of covalently bonded organic-inorganic hybrids were described. Molecular and polymeric hybrids based on covalently linked POMs and transition metal complexes have as of yet been limited to $\mathrm{Mo}_{6} \mathrm{O}_{19-x}(\mathrm{NR})_{x},(x=1$ or 2$)$ and cluster type POMs can also be linked through direct condensation or secondary metal ligand coordination complexes. Other examples of POM polymers, covalent hybrid materials based on nanolatex particles and Dawson clusters were reported recently (Cannizzo et al 2005), while coordination polymers with controllable growth of chains and grids from polyoxomolybdate building blocks were demonstrated by several workers. The most stable product in the polymerization of metal oxo-buildings is the metal oxide itself. Generally, the approaches used to produce high nuclearity POM-based clusters are extremely simple, consisting of an acidifying aqueous solution containing the relevant metal 
oxide anions. In case of acidification, for example, a solution of sodium molybdate will give rise to metal oxide fragments, which increases in nuclearity as the $\mathrm{pH}$ of the solution decreases (Pope 1983).

Since most of the applications of POMs require high surface area, nanotechnology can play a major role in the synthesis of such POMs. However, chemically synthesized POMs are still almost in the range of a few nanometers; it is difficult to control their size and morphology (Roy et al 2008). Hence, there is an immediate need to develop design principles that allow easy control of POM morphology and size on macroscopic length scales. At this point, it is worth mentioning that a significant development in the field of POMs was achieved when complex molecular POMs were self-assembled as large hollow spheres of 50-100 nm (Liu and Liu 2005). At present, many different nanomaterials of POMs such as nanoparticle, nanorod, nanowire, nanotube and nanohollow on the film have been prepared (Ting et al 2009). In the present work, we made an attempt to design a method to synthesize nanospheres of SMO in the range of 10-100 nm under ordinary conditions from aqueous solution by satisfying the major requirements of green chemistry.

\section{Experimental}

\subsection{Synthesis of SMO}

All chemicals are of reagent grade and were used without any further purification. In the present work, sodium molybdate as molybdenum source, DPC as structure directing template and double-distilled water as the medium of reactions were used. The synthesis of SMO was carried out by preparing two different solutions. $\mathrm{Na}_{2} \mathrm{MoO}_{4} \cdot 2 \mathrm{H}_{2} \mathrm{O}$ solution was slowly added to DPC in $1 \mathrm{M}$ solution of $\mathrm{H}_{2} \mathrm{SO}_{4}$ with mild stirring to avoid lather formation by keeping the mass ratio as $\mathrm{DPC} / \mathrm{Na}_{2} \mathrm{MoO}_{4} \cdot 2 \mathrm{H}_{2} \mathrm{O}=0 \cdot 33$, a yellow green precipitate was formed at $\mathrm{pH} 2.5$, which was adjusted to $\mathrm{pH} 1.5$ by adding $\mathrm{H}_{2} \mathrm{SO}_{4}$. The above mixture was then stirred continuously for $24 \mathrm{~h}$ (Asnani et al 2007; Thomas et al 2008a, b). The precipitate was washed continuously with doubledistilled water and then with absolute alcohol thoroughly to remove the excess surfactant. The whole process was carried out at room temperature without using any energy source. The formed precipitate was then dried below $323 \mathrm{~K}$. Another sample of SMO was also prepared through the same reaction process by keeping $24 \mathrm{~h}$ stirred mixture undisturbed to $48 \mathrm{~h}$ for aging.

\subsection{Characterization}

FT-IR spectrum was recorded on $\mathrm{KBr}$ pellets using Schimadzu spectrophotometer. ICP-AES analysis results were obtained from thermo-electron IRIS interprid 11 XSP DUO with a detection limit in ppm. TGA was carried out using Universal V3.9A TA instrument at a heating rate of $20{ }^{\circ} \mathrm{C} / \mathrm{min}$ in $\mathrm{N}_{2}$ atmosphere $(50 \mathrm{~mL} / \mathrm{min})$. The size and morphology of the samples were analysed using 1144SE1 for SEM and energy dispersive X-ray analysis (EDAX) measurements. TEM studies were conducted by Philips CM-20 electron microscope operated at $100 \mathrm{kV}$. Before TEM measurements, the sample was sonicated in absolute alcohol for $45 \mathrm{~min}$ and then deposited on carbon-coated copper grids. AFM studies were performed by scanning probe microscope (CP Research, Thermo microscope, USA). The topographs were collected in non-contact mode, where cantilever was vibrated at its resonance frequency of $320 \mathrm{kHz}$. Deviation from the resonance frequency was tracked with the help of a link-in amplifier, which formed the topographical data set. AFM images were recovered by dropping the specimen solution in absolute alcohol on silicon substrate with subsequent drying.

\subsection{Gravimetric estimation of sulphate}

A definite mass of SMO was dissolved in $100 \mathrm{~mL}$ of $5 \mathrm{M}$ $\mathrm{NaOH}$ solution. $20 \mathrm{~mL}$ of the solution was pipetted out and acidified with a solution of $\mathrm{HCl}$. It was diluted, warmed and treated with $\mathrm{BaCl}_{2}$ solution for complete precipitation. The white crystalline precipitate of $\mathrm{BaSO}_{4}$ was repeatedly washed with $\mathrm{HCl}$ and then with distilled water until the washings were free from $\mathrm{Cl}^{-}$ions. The dried $\mathrm{BaSO}_{4}$ was weighed for determination of sulphate content.

\section{Results and discussion}

\section{$3.1 \quad F T-I R$ analysis}

FT-IR spectrum of the synthesized sample of SMO is depicted in figure 1. An intense and wide peak centred at $3290 \mathrm{~cm}^{-1}$ is due to O-H stretching vibrations of adsorbed water. The peaks at 2883,2806 and $2723 \mathrm{~cm}^{-1}$ are attributed to aliphatic $\mathrm{C}-\mathrm{H}$ vibrations of DPC. Peaks at 1655 and $1568 \mathrm{~cm}^{-1}$ are assigned as $\mathrm{C}-\mathrm{H}$ extending vibrations while those at 1473,1383 and $1348 \mathrm{~cm}^{-1}$ correspond to $\mathrm{N}-\mathrm{H}$ extending vibrations of DPC moiety. The peaks centred at 1200 and $1074 \mathrm{~cm}^{-1}$ correspond to asymmetric and symmetric stretching of $\mathrm{SO}_{4}$ groups, respectively. The sharp peaks around 978,860 and 764 are attributed to $\mathrm{Mo}=\mathrm{O}$ and $\mathrm{Mo}-\mathrm{O}$ vibrations. The peak centred at $696 \mathrm{~cm}^{-1}$ confirms the presence of Mo-O-S lattice. The peaks at 632 and $503 \mathrm{~cm}^{-1}$ are due to asymmetric and symmetric bending vibrations of $\mathrm{SO}_{4}$ group of SMO.

\subsection{SEM, TEM and AFM analysis}

Figure 2 displays SEM image of SMO nanospheres. It shows a large extent of clustering due to weak attractive forces like Van der Waal's forces between the particles. Therefore, we found it necessary to sonicate the particles for a few min before taking the electron photographs of SMO.

Figure 3(a) shows TEM image of synthesized sample of SMO without aging, where the diameter of nanospheres are 


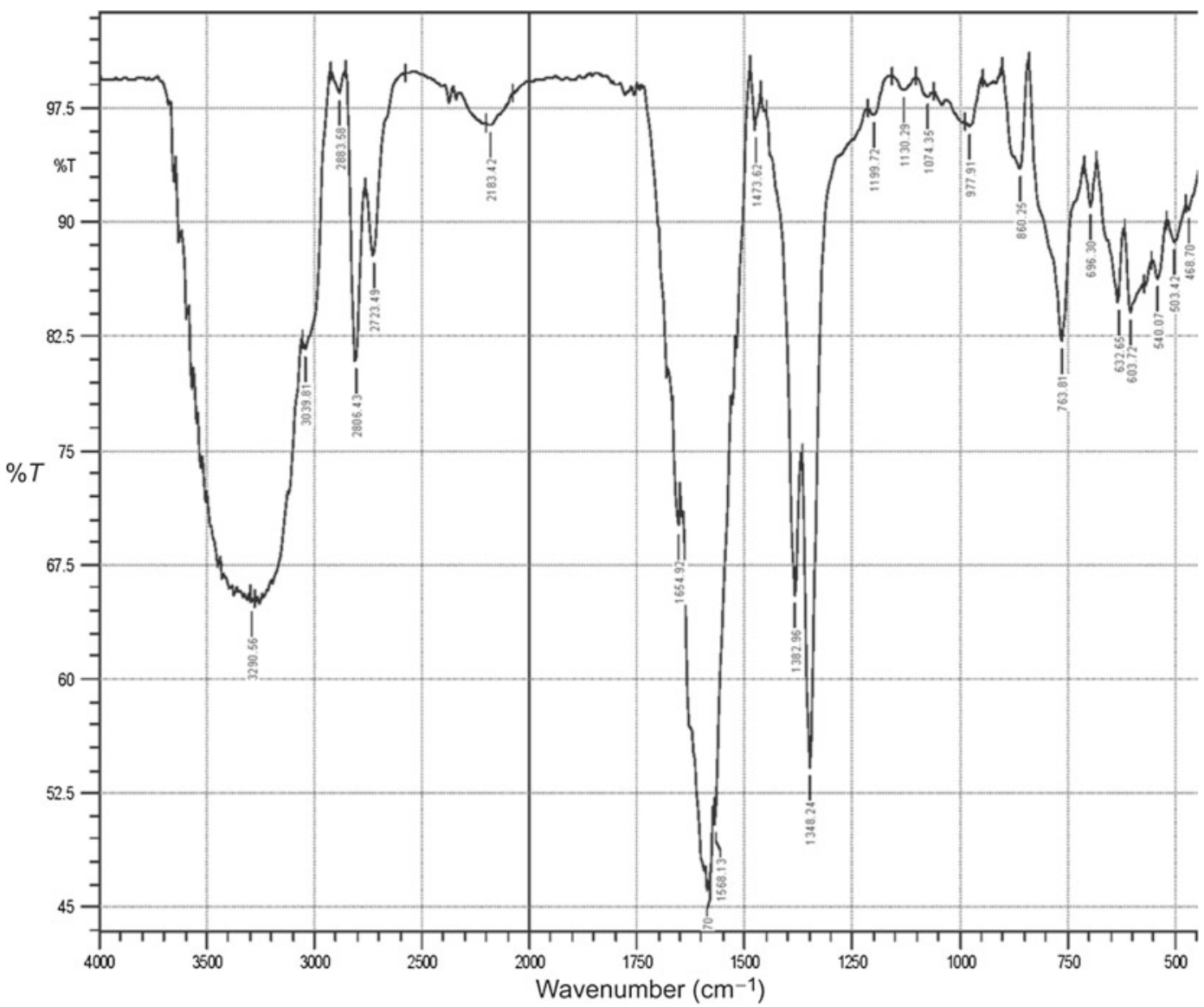

Figure 1. FT-IR spectrum of sample of SMO recorded on $\mathrm{KBr}$ pellets.

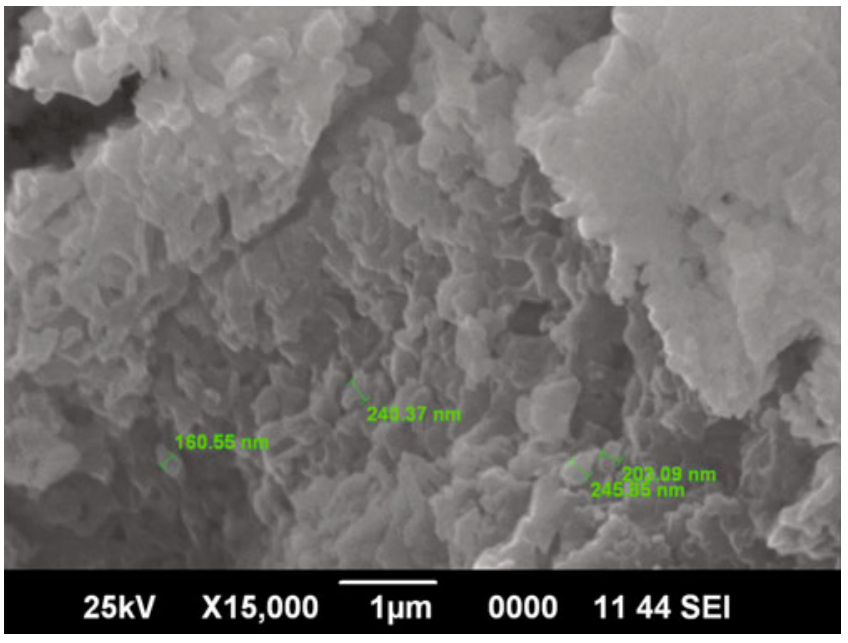

Figure 2. SEM image of SMO obtained without sonication of sample.

mostly in the range of 10-25 nm. Figure 3(b) is TEM image of SMO obtained after aging for $48 \mathrm{~h}$ and shows the presence of larger particles having diameter in the range of 40-75 nm. This is attributed to the fact that clustering leads to enhancement of particle size which occurs on aging of the sample (Swarnkar et al 2011). Figure 3(c) displays magnified TEM image of SMO obtained after aging for $48 \mathrm{~h}$ and it reveals the presence of multiple twinned particles (MTPs) with a size $>40 \mathrm{~nm}$. These large-sized MTPs are formed by the joining of a few clusters of SMO particles with size $<25 \mathrm{~nm}$ joined through twin boundaries. These structures do not form completely space-filling structures and require twin boundaries to do so.

Figure 4(a-c) shows AFM images of SMO nanospheres, islands and sheets, respectively. Figure 4(a) displays AFM image of nanospheres of SMO around $50 \mathrm{~nm}$. In figure 4(b), there are small islands of SMOs of height $40-100 \mathrm{~nm}$ consisting of MTPs. The formation of large size particles resulting from the clustering of small SMO particles are also observed in figure 4(c).

It is evident from all the electron and optical microscopic investigations carried out in the present work that there is formation of nanospheres of SMO with spherical shape. These spheres have diameters ranging from 20 to $100 \mathrm{~nm}$ with the highest population of particles around $50 \mathrm{~nm}$.

\subsection{Molecular formula of SMO}

Figure 5 shows thermogravimetric curve for the thermal decomposition of SMO in the temperature range of 310 $1100 \mathrm{~K}$ in $\mathrm{N}_{2}$ atmosphere $(50 \mathrm{~mL} / \mathrm{min})$. There is a small weight loss $(<5 \%)$ up to the temperature $573 \mathrm{~K}$ due to the 

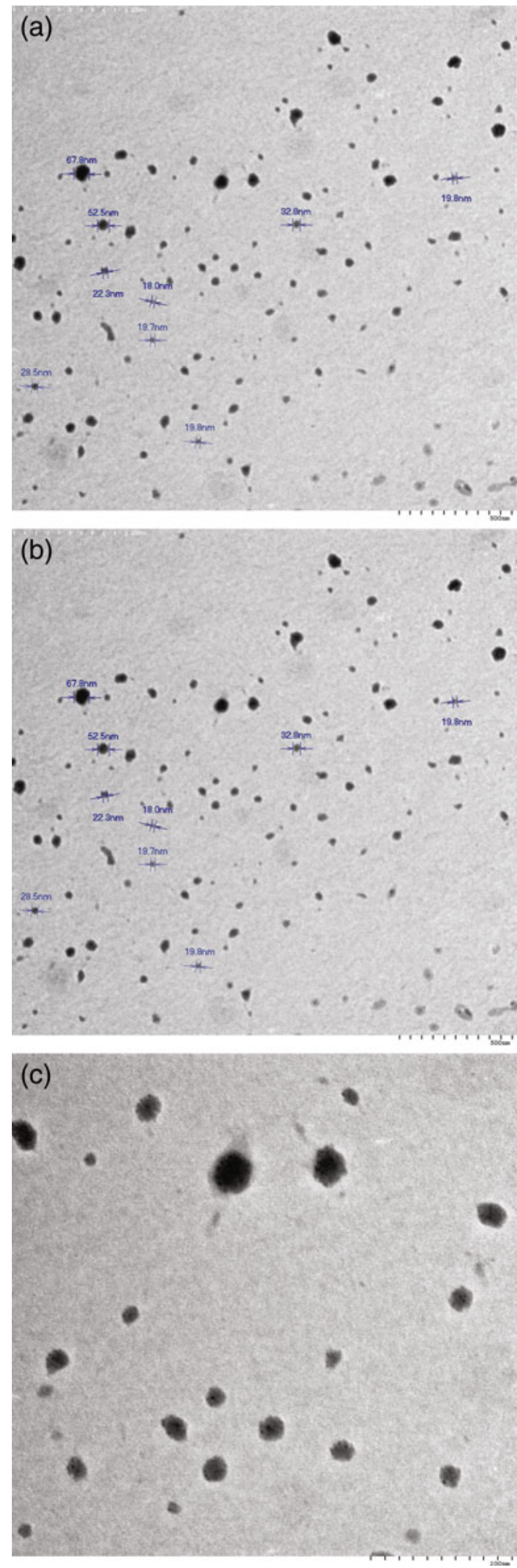

Figure 3. TEM image of SMO obtained by mixing reagents, stirred for $24 \mathrm{~h}$ and then filtered (a) without aging, (b) after $48 \mathrm{~h}$ of aging and (c) magnified TEM image of aged sample of SMO.

removal of surface adsorbed water molecules. There is a large decrease in mass $\approx 36 \%$ from 600 to $623 \mathrm{~K}$ due to the removal of DPC moiety incorporated in SMO framework. There is a third weight loss of about $6 \%$ from 673 to $973 \mathrm{~K}$ due (a)

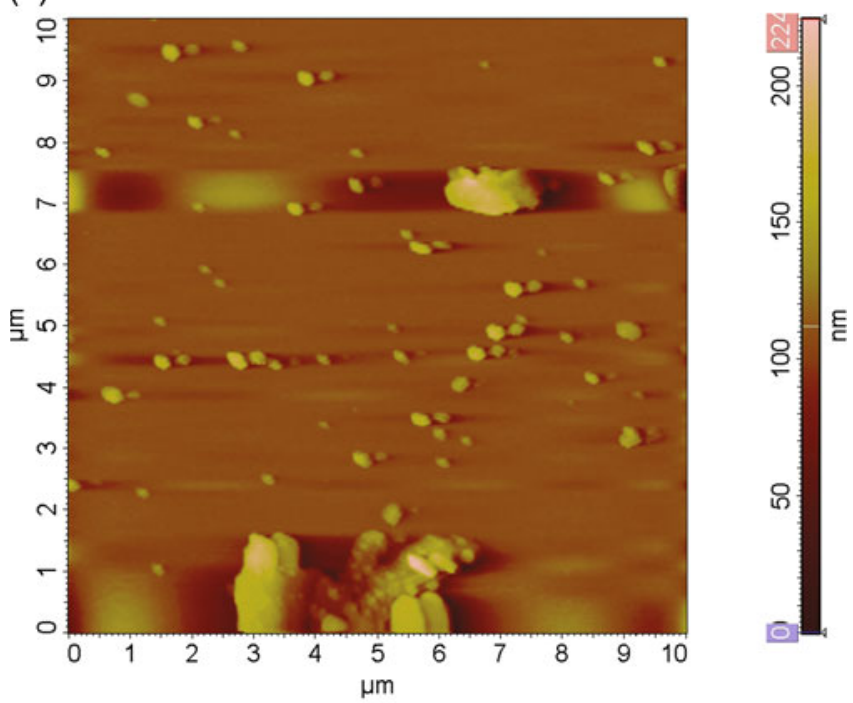

(b)

(c)
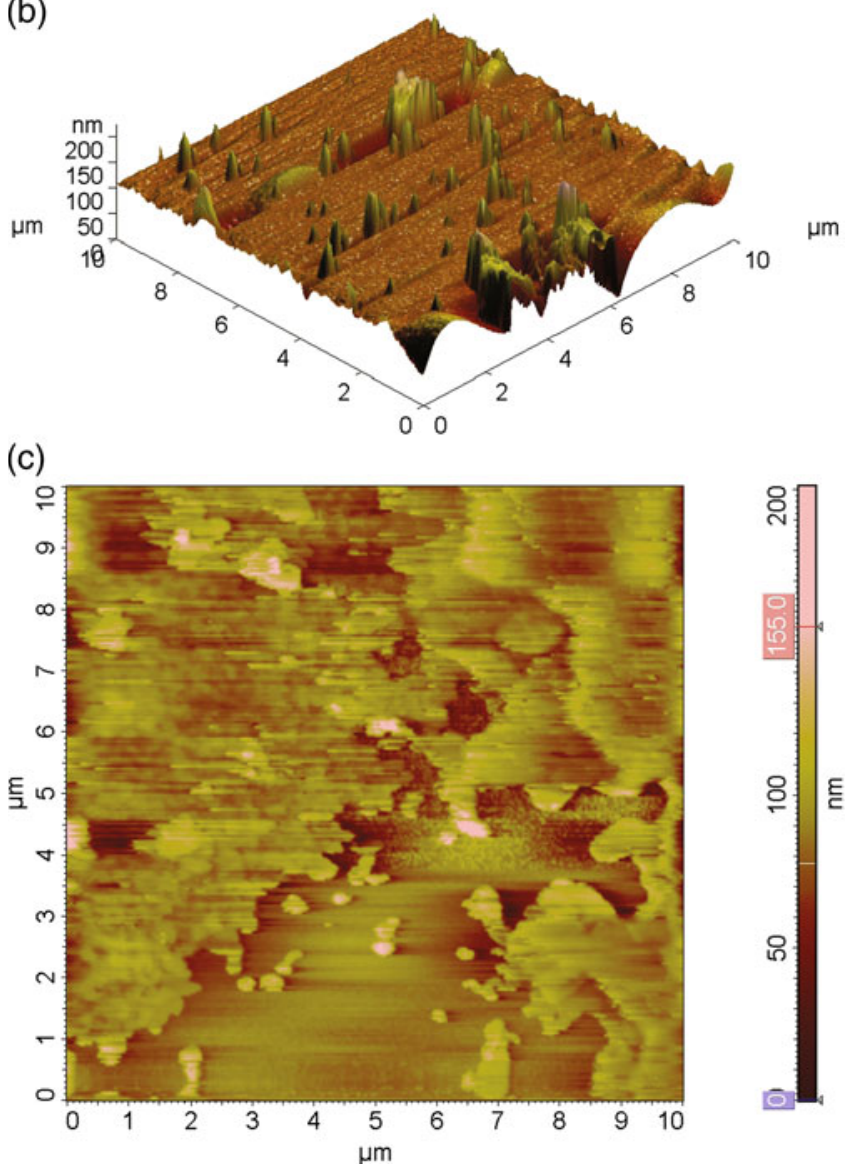

Figure 4. (a) AFM image of nanospheres of SMO obtained by dispersing in absolute alcohol, (b) AFM image of sample of SMO containing islands made up of multiple twinned particles and (c) AFM image of sample of SMO; lower part of figure shows small nanospheres of SMO and upper part shows large sheets of SMO resulting from clustering of small spheres.

to the removal of $\mathrm{SO}_{2}$ and thereafter the peak remains constant due to the stable end product, $\mathrm{MoO}_{3}$. ICP-AES analyses shows the presence of S in SMO. Based on TGA and 


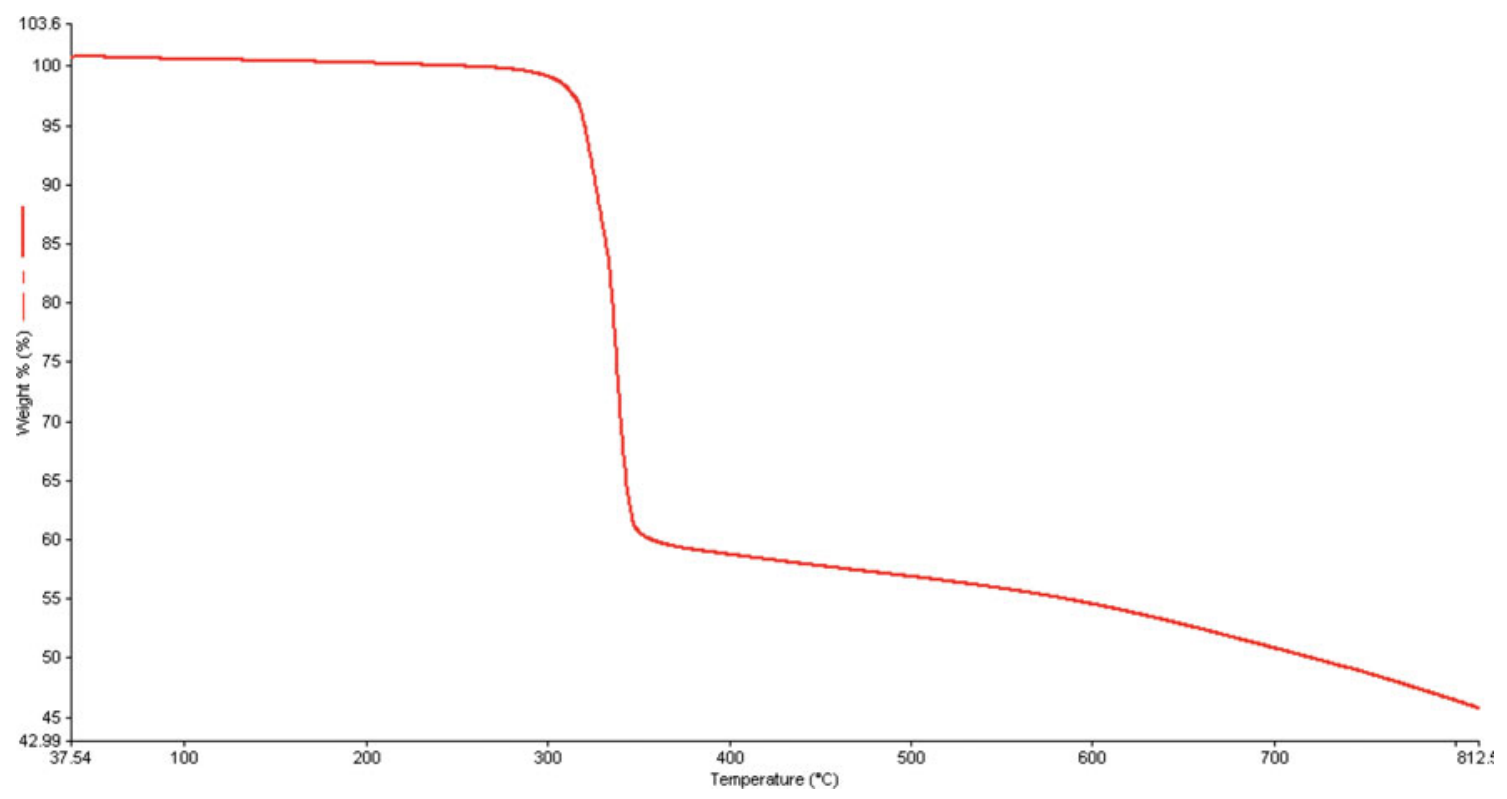

Figure 5. TGA of $\mathrm{SMO}$ at a heating rate of $20^{\circ} \mathrm{C} / \mathrm{min}$ in $\mathrm{N}_{2}$ atmosphere.

ICP-AES analyses, the approximate molecular formula of $\mathrm{SMO}$ is found to be $\mathrm{DPC}_{6}\left[\mathrm{SMo}_{10} \mathrm{O}_{35}\right] x \mathrm{H}_{2} \mathrm{O}(x<1)$. The quantity of sulphate determined gravimetrically is found to be in good agreement with this formula.

\subsection{Mechanism of formation of SMO nanospheres}

Crystallization or precipitation of a solid is essentially a self-assembly process. In aqueous solution, molybdate ions undergo continuous hydrolysis and condensation leading to a range of oligomeric anionic species; one or more of these selfassemble along with templates or metal ions or both (Patzke et al 2002; Thomas et al 2008a, b). The self-assembling process of molybdate among themselves and sulphate ions in presence of template DPC leads to nanolevel heteropolyanion of SMO. This can be seen from TEM and AFM analyses.

Nanospheres of SMO are produced by the electrostatic stabilization of negatively charged SMO by protonated DPC under acidic conditions. The single DPC incorporated SMOs undergo clustering to form MTPs with twin boundaries. MTPs have weaker bonding in twin boundary areas and can provide sites for further agglomeration, leading to the growth of nanospheres to other morphologies as shown in figure $6(\mathrm{a}$ and $\mathrm{b})$. There are reports for the coalescence of smaller nanostructures to larger nanoparticles on aging (Jana et al 2001; Pacholski et al 2002). The role of aging is, thus, to provide more time for the particles to undergo diffusion to form larger-dimensioned structures. Growth of nanospheres beyond micro level are terminated due to the thick multilayer formation of DPC around each particle and prevents further agglomeration as is evident from figures 3 and 6 in TEM and AFM analyses.

\subsection{Green synthetical importance of nano SMO formation}

Influence of reaction parameters such as temperature, surfactant (DPC) and $\mathrm{pH}$ of reaction medium in the formation of nano SMO were studied in the context of the requirements of green chemistry and are discussed here.

3.5a Effect of temperature: Reactions are influenced by temperature due to their exothermic or endothermic nature. The effect of temperature in the formation of nano SMO is governed by the thermodynamic expression:

$$
\ln K=\frac{\Delta S^{0}}{R}-\frac{\Delta H^{0}}{R T},
$$

where $K$ is the equilibrium constant, $\Delta S^{0}$ the standard entropy change, $\Delta H^{0}$ the standard enthalpy change, $R$ the universal gas constant and $T$ the temperature in Kelvin. For an endothermic reaction, $\Delta H^{0}$ is positive, an increase in temperature will increase the value of $K$. But for an exothermic reaction, the value of $K$ can be increased by lowering the temperature. Effect of temperature in the formation of SMO is illustrated in figure 7. It is seen that the formation of SMO is an endothermic reaction. However, the increment in the yield of SMO with increase in temperature is not so pronounced in the range of higher temperatures.

From the above equation, it follows that an increase in temperature and entropy will favour the formation of products with high yield. It is observed that with increase in number of particles of nanosize, the entropy also increases from quantum mechanical considerations. But an increase in temperature above ordinary temperature results in the agglomeration of particles beyond nano level, which is not advisable to the goal of the present work and is also contradictory to the requirements of green chemical path. But the use of energy 
(a)

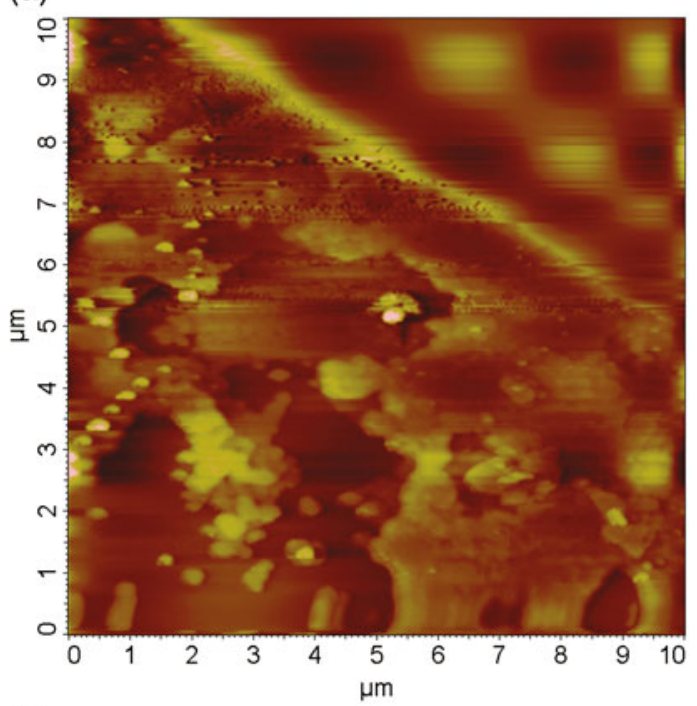

(b)

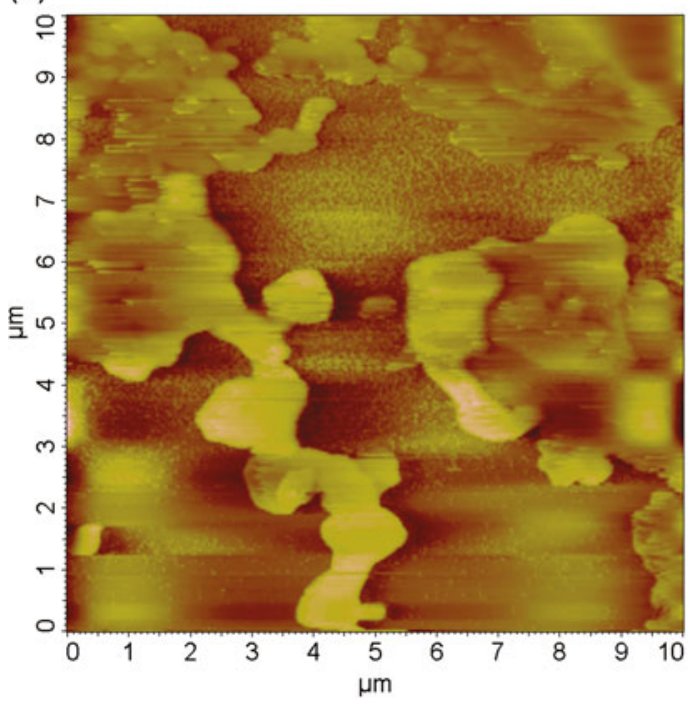

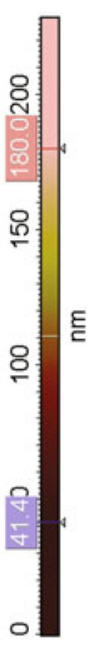

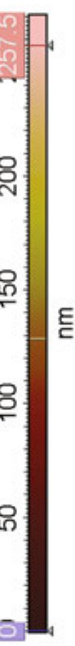

Figure 6. (a) AFM image of small nanospheres of SMO without aging. (b) AFM image of sample of SMO formed due to clustering of small nanoparticles after aging of $48 \mathrm{~h}$.

source for higher temperature is compensated by an increase of $\Delta S$, which results from the production of particles to the nano level with maximum population that gives a shake hand to green chemistry.

3.5b Effect of surfactant: POM syntheses are reported by using different surfactants and the products formed are of different morphologies. The difference in morphology is attributed to the difference in the number of carbon atoms in the templating agent. Our attempt to prepare SMO spheres by mixing the reagents without surfactant gave poor results and the product is either less stable or soluble in the reaction medium. Therefore, the use of surfactant DPC has played various significant roles in the preparation of $\mathrm{SMO}$, such as to control their dimensions to nano level, to get maximum yield

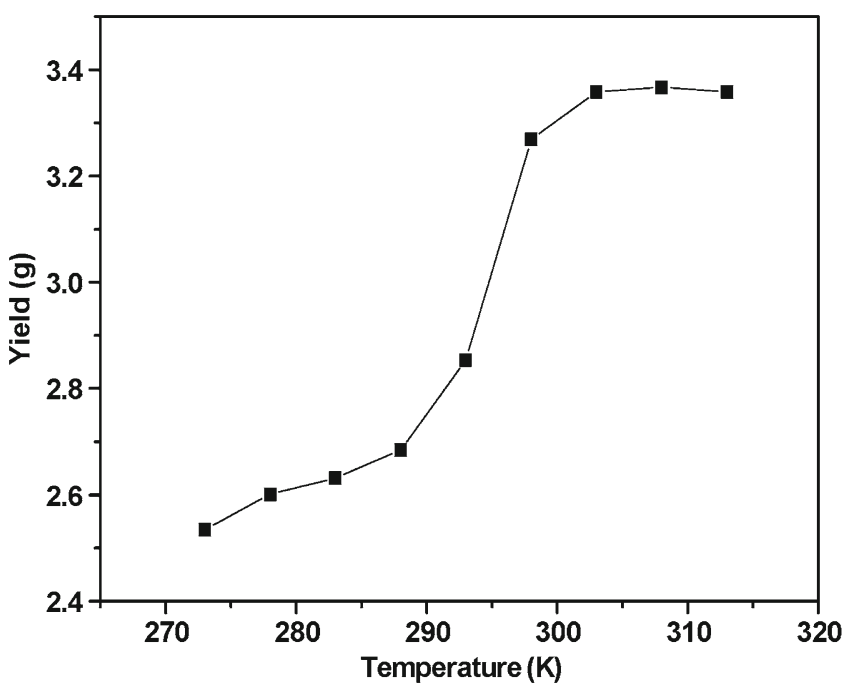

Figure 7. Effect of temperature in yield of SMO.

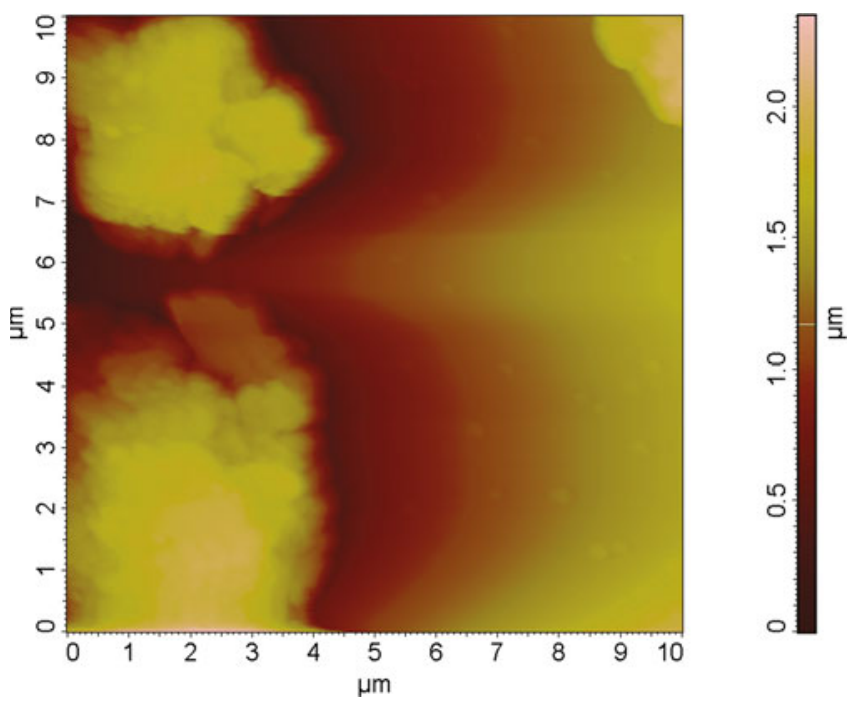

Figure 8. AFM image of lamellar or micellar structure of SMO obtained for mass ratio of DPC $/ \mathrm{Na}_{2} \mathrm{MoO}_{4} \cdot 2 \mathrm{H}_{2} \mathrm{O}=0.5$.

and to make them stable. The protonated DPC is an alternative to other poisonous metal ions that are used to stabilize POMs and is an approach to green chemistry. A remarkable amount of nanospheres are obtained to an optimum mass ratio of $\mathrm{DPC} / \mathrm{Na}_{2} \mathrm{MoO}_{4} \cdot 2 \mathrm{H}_{2} \mathrm{O}=0 \cdot 33$, but increasing the mass ratio from 0.33 to 0.5 results in the formation of lamellar or micellar structures as shown in figure 8 .

3.5c Effect of $\mathrm{pH}$ of reaction medium: Effect of $\mathrm{pH}$ in the formation of SMO is shown in figure 9. It is seen that the yield of SMO is more in the $\mathrm{pH}$ range of 1.5-2.5 and there is no precipitate below $\mathrm{pH} 0.5$ and above $\mathrm{pH} 6$. At $\mathrm{pH}<0.5$, $\mathrm{H}^{+}$ions can replace DPC and form protonated soluble polyoxoacid of SMO, whereas at $\mathrm{pH}>6$, DPC is set free by the attack of corresponding base, causing POM soluble in the 


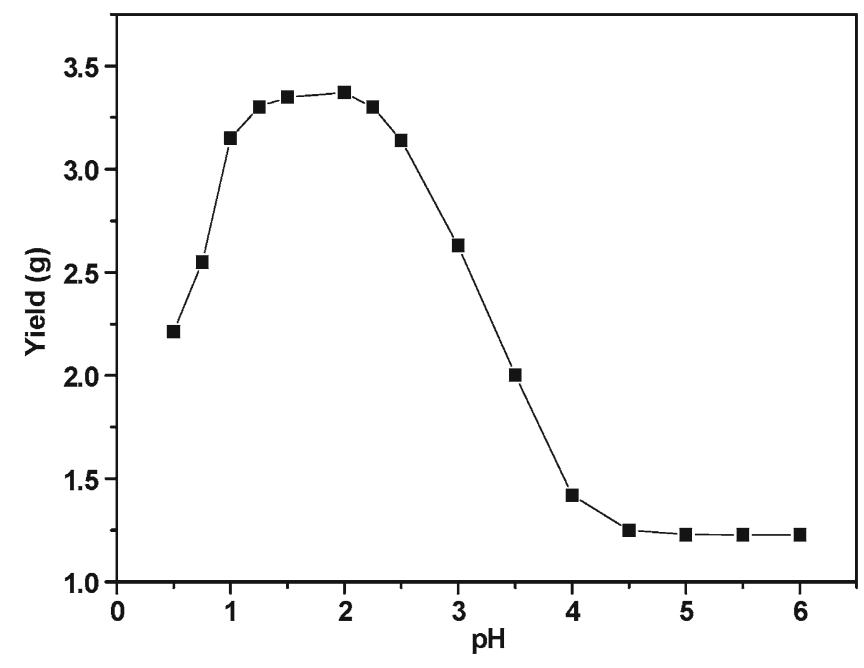

Figure 9. Effect of $\mathrm{pH}$ in formation of nano SMO.

reaction medium. However, through the windows of green chemistry, the precipitation under acidic $\mathrm{pH}$ is not a good one. However, if we make use of most of the industrial wastes of acidic $\mathrm{pH}$ range, for the precipitation of SMO or similar POMs for various processes like catalysis, adsorption, etc it will become a promising alternative for environmental problems.

\section{Importance of nano SMO in material science}

The synthesized nano SMO is a pale-yellow green coloured amorphous material with thermal stability up to $600 \mathrm{~K}$. It is insoluble in water, alcohol, acetone, ether, etc and can withstand up to $\mathrm{pH}=8$. These characteristics make nano SMO suitable for applications in various areas of research like water treatment and catalysis. The green synthetic path of SMO is of great interest in our industrialized century for ecological and environmental reasons.

\section{Conclusions}

The present work deals with the synthesis and characterization of SMO nanospheres. Synthesis under suitable conditions based on requirements of green chemistry using DPC as template led to the formation of SMO nanospheres with the highest population between 25 and $50 \mathrm{~nm}$. It is one of the best methods compared to other methods, like hydrothermal and microwave-assisted synthesis, which are not suited for the eco-friendly operations and cost saving. The thermal stability, insolubility in various reaction mediums, stability towards a wide range of $\mathrm{pH}$, etc will make a significant and excellent effect on its applications. The mechanisms of formation of large spheres of SMO from small nanoparticles and its further results related to thermodynamic parameters give a better insight into the growth of nano POMs improvement of synthetic strategies, control of particles to different morphologies and other future applications.

\section{Acknowledgements}

The authors are grateful to the University Grants Commission for the financial assistance and we wish to express our thanks to NIIST, Thiruvananthapuram; STIC, Cochin and SCTIMST, Thiruvananthapuram; for lending the analytical facilities.

\section{References}

Asnani M, Thomas J, Sen P and Ramanan A 2007 Mater. Res. Bull. 42686

Cannizzo C, Mayer C R, Secheresse F and Carpent C 2005 Adv. Mater. 172888

Jana N R, Gearheart C and Murphy C J 2001 Adv. Mater. 131389

Kang J, Xu B B, Peng Z H, Zhu X D, Wei Y G and Powell D R 2005 Angew. Chem. Int. Ed. 446902

Liu G and Liu T 2005 Langmuir 212713

Liu T B, Diemann E, Li H L, Dress A W M and Muller A 2003 Nature $\mathbf{4 2 6} 59$

Long D L, Burkholder E and Cronin L 2007 Chem. Soc. Rev. 36105

Muller A et al 2002 Angew. Chem. Int. Ed. 41579

Pacholski C, Kornowski A and Weller H 2002 Angew. Chem. Int. Ed. 411188

Patzke G R, Krumeich F and Nesper R 2002 Angew. Chem. Int. Ed. 412446

Pope M T 1983 Heteropoly and isopoly-oxo metalates (Berlin: Springer Verlag)

Roy S, Bossers L C A M, Meeldijk H J D, Kuippers B W M and Kegel W K 2008 Langmuir 24666

Sanghi R and Srivastava M M 2007 Green chemistry environment friendly alternatives, eds R Sanghi and M M Srivastava (New Delhi: Narosa Publishing House Pvt. Ltd.)

Swarnkar R K, Singh S C and Gopal R 2011 Bull. Mater. Sci. 34 1363

Thomas J, Asnani M, Sharma S and Ramanan A 2008a Nanostructure vanadium oxides in encyclopedia of nanoscience and nanotechnology (USA: American Scientific Publishers)

Thomas J, Kannan K R and Ramanan A 2008b J. Chem. Sci. 120 529

Ting L W, Chengzhi H and Lu M A 2009 Sci. China Ser. B-Chem. 522156 